\title{
BMJ Open Assessing the safety, tolerability and efficacy of PLGA-based immunomodulatory nanoparticles in patients with advanced NY-ESO-1- positive cancers: a first-in-human phase I open-label dose-escalation study protocol
}

\author{
Jeroen H A Creemers (D) , ${ }^{1,2}$ Inka Pawlitzky, ${ }^{3}$ Konstantina Grosios, ${ }^{4}$ Uzi Gileadi, \\ Mark R Middleton, ${ }^{6}$ Winald R Gerritsen, ${ }^{7}$ Niven Mehra, ${ }^{7}$ Licia Rivoltini, ${ }^{8}$ \\ Ian Walters, ${ }^{9}$ Carl G Figdor, ${ }^{1,2}$ Petronella B Ottevanger, ${ }^{7}$ I Jolanda M de Vries ${ }^{1}$
}

To cite: Creemers JHA, Pawlitzky I, Grosios K, et al. Assessing the safety, tolerability and efficacy of PLGA-based immunomodulatory nanoparticles in patients with advanced NY-ES0-1-positive cancers: a first-in-human phase I open-label dose-escalation study protocol. BMJ Open 2021;11:e050725. doi:10.1136/ bmjopen-2021-050725

- Prepublication history and additional supplemental material for this paper are available online. To view these files, please visit the journal online (http://dx.doi.org/10.1136/ bmjopen-2021-050725).

IP and KG contributed equally. CGF and PBO contributed equally.

Received 26 February 2021 Accepted 22 0ctober 2021

\section{Check for updates}

(c) Author(s) (or their employer(s)) 2021. Re-use permitted under CC BY-NC. No commercial re-use. See rights and permissions. Published by BMJ.

For numbered affiliations see end of article.

\section{Correspondence to} Dr I Jolanda M de Vries; Jolanda.devries@radboudumc. $\mathrm{nl}$

\section{ABSTRACT}

Introduction The undiminished need for more effective cancer treatments stimulates the development of novel cancer immunotherapy candidates. The archetypical cancer immunotherapy would induce robust, targeted and long-lasting immune responses while simultaneously circumventing immunosuppression in the tumour microenvironment. For this purpose, we developed a novel immunomodulatory nanomedicine: PRECIOUS-01. As a PLGA-based nanocarrier, PRECIOUS-01 encapsulates a tumour antigen (NYESO-1) and an invariant natural killer T cell activator to target and augment specific antitumour immune responses in patients with NY-ESO-1-expressing advanced cancers.

Methods and analysis This open-label, first-inhuman, phase I dose-escalation trial investigates the safety, tolerability and immune-modulatory activity of increasing doses of PRECIOUS-01 administered intravenously in subjects with advanced NY-ESO-1expressing solid tumours. A total of 15 subjects will receive three intravenous infusions of PRECIOUS-01 at a 3-weekly interval in three dose-finding cohorts. The trial follows a $3+3$ design for the dose-escalation steps to establish a maximum tolerated dose (MTD) and/or recommended phase II dose (RP2D). Depending on the toxicity, the two highest dosing cohorts will be extended to delineate the immune-related parameters as a readout for pharmacodynamics. Subjects will be monitored for safety and the occurrence of dose-limiting toxicities. If the MTD is not reached in the planned dose-escalation cohorts, the RP2D will be based on the observed safety and immune-modulatory activity as a pharmacodynamic parameter supporting the RP2D. The preliminary efficacy will be evaluated as an exploratory endpoint using the best overall response rate, according to Response Evaluation Criteria in Solid Tumors V.1.1.

\section{Strengths and limitations of this study}

This is the first-in-human study with a novel class of off-the-shelf immunomodulatory nanomedicines: PLGA nanoparticles encapsulating tumor-antigen NY-ES0-1 and invariant natural killer T (iNKT) cell activator IMM60 can be administered intravenously, are versatile and induce multifunctional antitumour immune responses compared with cellular immunotherapies.

- Coencapsulation of a tumor antigen and an iNKT cell activator leads to optimal activation and antigen presentation of dendritic cells and provides a boost by bridging innate and adaptive immunity.

- A recommended phase II dose based on safety/tolerability endpoints and local (tumour biopsies) as well as systemic immune-modulatory assessments ensures the selection of a safe and biologically meaningful dose for later phase clinical trials.

- Although cancer germline antigen NY-ESO-1 is expressed in a wide variety of advanced cancers, it is not a neoantigen, and the potential for an antitumour immune response of PRECIOUS-01 may be limited in NY-ESO-negative tumours.

- This first-in-human phase I trial is not powered for efficacy endpoints and is therefore only suited to gather preliminary efficacy data.

Ethics and dissemination The Dutch competent authority (CCMO) reviewed the trial application and the medical research ethics committee (CMO ArnhemNijmegen) approved the trial under registration number NL72876.000.20. The results will be disseminated via (inter)national conferences and submitted for publication to a peer-reviewed journal. Trial registration number NCT04751786. 


\section{INTRODUCTION}

Despite revolutionising progress in immunotherapy for advanced solid tumours, there is an undiminished need to develop immunotherapies that can orchestrate an effective antitumour immune response in a highly targeted way while simultaneously circumventing immunosuppression in the tumour microenvironment. To this end, we developed a novel immunomodulatory nanomedicine: PRECIOUS-01. It consists of a poly (lactic-co-glycolic acid) (PLGA)-nanoparticle-based carrier platform that encapsulates tumour antigen New York Esophageal Squamous Cell Carcinoma-1 (NY-ESO-1) and an invariant natural killer T (iNKT) cell activator (ie, threitolceramide-6, hereafter called IMM60) and aims to induce a specific antitumour immune response.

As a drug-carrying platform, PLGA has two main advantages: it is biodegradable and elicits minimal systemic toxicity. ${ }^{1}$ Nanoparticle-encapsulated antigens are taken up more efficiently by dendritic cells (DCs) than their soluble counterparts. ${ }^{2}$ Subsequent prolonged presentation of PLGA-encapsulated peptides on major histocompatibility complex classes I and II molecules can generate functional and tumor-antigen-specific $\mathrm{CD}^{+}$and $\mathrm{CD}^{+}$ T-cell responses in preclinical studies. ${ }^{3}$ Moreover, given their rapid internalisation by myeloid cells after systemic injection, PLGA nanoparticles contribute to perturb macrophage polarisation and reprogramming within the tumour microenvironment, thus establishing the proper milieu for immune response induction. ${ }^{4}$ For an extensive overview of immunomodulatory nanoparticles in cancer immunotherapy, we refer to a review by Feng $e t a .^{5}$

The first encapsulated component in PRECIOUS-01, functioning to target the immune response, is the cancertestis antigen NY-ESO-1. While absent on normal somatic tissue, NY-ESO-1 is expressed on many advanced cancers: lung $(2 \%-32 \%)$, melanoma $(40 \%)$, bladder $(32 \%-35 \%)$, prostate $(38 \%)$, ovarian $(30 \%)$, oesophageal $(24 \%-33 \%)$, breast $(12 \%-28 \%)$ and gastric $(8 \%-12 \%) \cdot{ }^{6-13}$ We defined two long peptides and one short peptide for nanoparticle encapsulation (85-111, 117-143 and 157-165), which combined cover more than $80 \%$ of the European population for both class I and class II Human Leukocyte Antigen (HLA) alleles. ${ }^{14}$ NY-ESO-1-specific peptides, produced according to Good Manufacturing Practices guidelines, are safe and tolerated in patients with cancer and have been shown to induce antigen-specific T-cell responses against NY-ESO-1-positive tumours. ${ }^{15-18}$

The second encapsulated component is the alphagalactosylceramide ( $\alpha$ GalCer)-derived iNKT cell activator IMM60. IMM60 is presented on CD1d molecules on DCs and possesses a superior iNKT cell activation profile compared with $\alpha$ GalCer in vitro as well as in vivo and is not subject to glycosidase-mediated degradation. ${ }^{19}$ On activation, iNKT cells secrete proinflammatory cytokines (eg, interferon- $\gamma$ (IFN- $\gamma$ ) and interleukin-12) and stimulate DC maturation in a CD40-CD40 ligand-dependent matter. ${ }^{20}$ iNKT cell-mediated antitumour effects include IFN- $\gamma$-dependent natural killer (NK) cell responses,

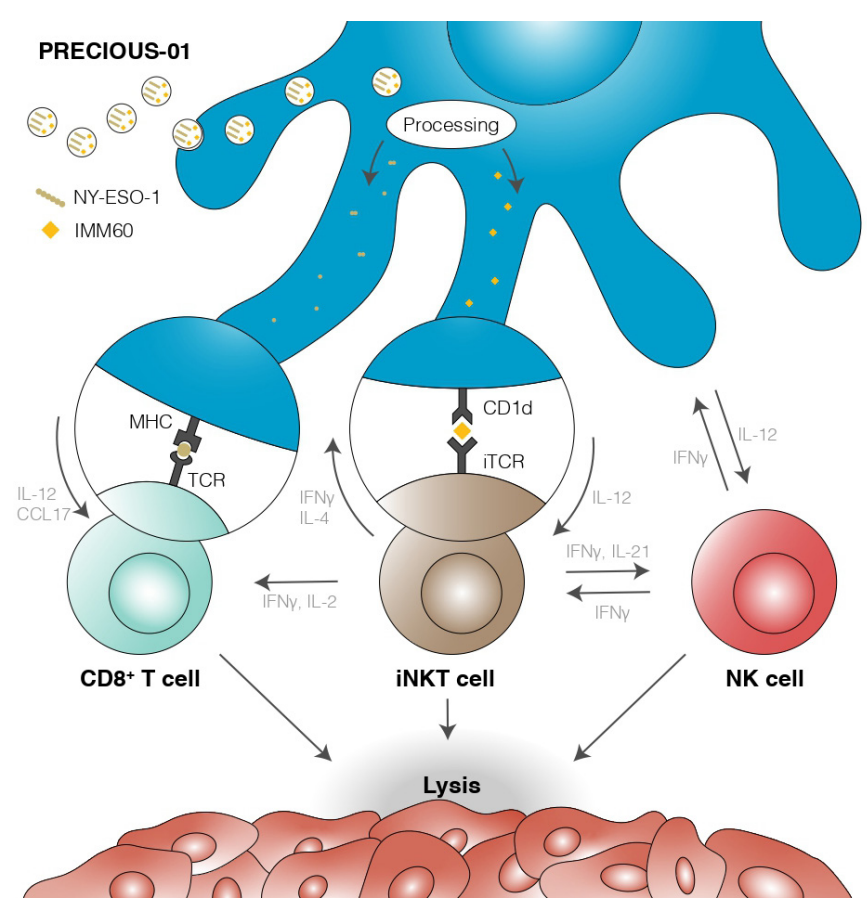

Figure 1 Overview of the mechanism of action of PRECIOUS-01. DCs take up and process PRECIOUS-01. NY-ESO-1 presentation on MHC I/II generates specific T-cell responses, while CD1d-dependent presentation of IMM60 on DCs leads simultaneously to iNKT cell activation and augmentation of the antitumour immune response, ultimately culminating in tumour cell killing. DCs, dendritic cells; IFN$\gamma$, interferon- $\gamma$; IL-12, interleukin-12; iNKT, invariant natural killer T cell; iTCR, invariant T-cell receptor; MHC, major histocompatibility complex; NK, natural killer; NY-ESO-1, New York Esophageal Squamous Cell Carcinoma-1.

expansion of antigen-specific CD8 ${ }^{+} \mathrm{T}$-cell responses, inhibition of metastatic behaviour and improved survival in cancer mouse models. ${ }^{190}$ Thus, with IMM60 as an adjuvant, we expect to boost the adaptive-immune and innateimmune response.

We hypothesised that coencapsulation of iNKT cell activator IMM60 with tumour antigen NY-ESO-1 results in detectable cytotoxic T-lymphocyte responses against the respective antigen. In vitro experiments with peripheral blood mononuclear cells demonstrated the feasibility of encapsulating three NY-ESO-1 peptides together with IMM60 in the same particle. ${ }^{21}$ Peptides were processed and presented by multiple HLA types. In mice expressing the human HLA-A2 molecule, the activity of nanoparticleencapsulated IMM60 was evident by DC maturation and IFN $\gamma$ secretion. $\mathrm{CD}^{+}$and $\mathrm{CD} 8^{+}$T-cell responses against NY-ESO-1 peptides were observed with the same nanoparticles coencapsulating NY-ESO-1 peptides. ${ }^{21}$ Preclinical pharmacology and toxicology studies indicated that PRECIOUS-01 was well tolerated and induced NY-ESO1 -specific antibodies. Furthermore, similar particles, in which ovalbumin was used as antigen, prolonged survival 
of murine cancer models. ${ }^{22}$ An overview of the mechanism of action of PRECIOUS-01 is provided in figure $1 .^{23-27}$

The purpose of this first-in-human phase I doseescalation trial is to determine the safety, tolerability and immune activity of increasing doses of PRECIOUS-01 in subjects with NY-ESO-1-expressing solid tumours. Threeweek intervals are placed between repeated administrations to provide time for recovery of the activated iNKT cells. The full study protocol is included as online supplemental information.

\section{METHODS AND ANALYSIS Study objectives}

The coprimary objectives of this trial are to (1) determine the safety and tolerability of increasing doses of PRECIOUS-01 after intravenous administration in subjects with NY-ESO-1-expressing solid tumours and (2) assess the effect of increasing doses of PRECIOUS-01 on the composition and spatial heterogeneity of immune cells directly in the tumour. The secondary objectives are to determine the recommended phase II dose (RP2D) of PRECIOUS-01 for a subsequent phase II trial and assess immunological responses during and after therapy with PRECIOUS-01 in blood. Furthermore, evaluation of the clinical outcome of PRECIOUS-01 using Response Evaluation Criteria in Solid Tumors (RECIST) V.1.1 is defined as an exploratory objective.

\section{Trial endpoints}

Primary endpoints

- Safety profiles: incidence of treatment-emerging adverse events (AEs) and serious AEs (SAEs) and laboratory abnormalities graded according to the National Cancer Institute Common Terminology Criteria for Adverse Events (CTCAE) V.5.0 reporting severity and relatedness; occurrence of dose-limiting toxicities (DLTs); incidence of treatment discontinuations and treatment modifications due to AEs and laboratory abnormalities; changes in vital signs, ECG and Eastern Cooperative Oncology Group performance status and deaths.

- The immune-modulating effect directly in the tumour will be assessed at baseline and after cycle 3 to analyse the immunological composition in tumour biopsies using an established immunohistochemistry (IHC) assay for the quantification of CD3, CD8, FoxP3, CD45RO and CD20-positive cells; these results will support the decision on the RP2D as part of the planned trial.

The secondary endpoints

- The RP2D, which will be evaluated using all data on safety and the immune-modulating effect per dose level tested (if maximum tolerated dose (MTD) is reached at one of the tested dose levels, then this dose will be used).

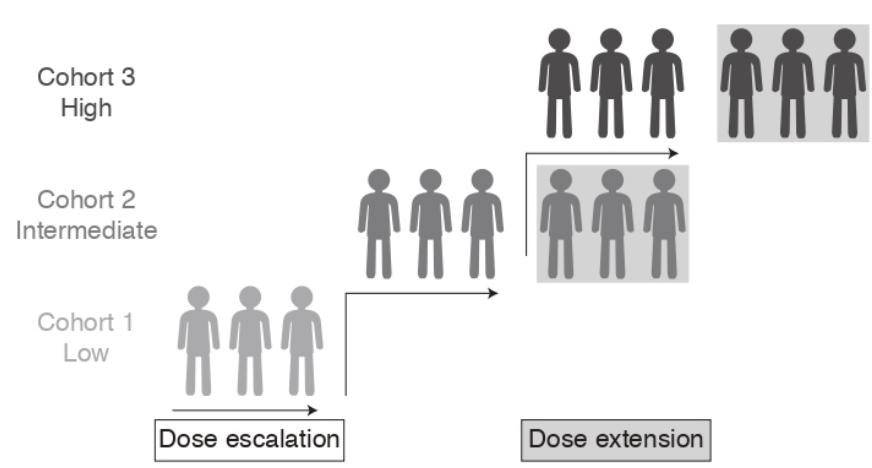

Figure 2 Trial design overview.

- Evaluation of the immune-modulating activity at each dose level (ie, the presence of NY-ESO-1-specific T cells, iNKT cell activation, DC activation, cytokine responses and anti-NY-ESO-1-specific antibody responses) using peripheral blood.

\section{Exploratory endpoint}

- Best overall response (BOR) according to RECIST V.1.1 at each dose level.

\section{Trial design}

This is an open-label, first-in-human, phase I doseescalation trial to investigate the safety and tolerability of increasing doses of PRECIOUS-01 administered intravenously in subjects with NY-ESO-1-expressing solid tumours. Enrolment has started on 11 January 2021 in Radboudumc, The Netherlands. The primary endpoint completion date and the study completion date are estimated to be September 2022 and December 2022, respectively. Figure 2 depicts the trials' design.

A planned total of 15 eligible subjects will receive three intravenous infusions of PRECIOUS-01 at a 3-weekly interval in three dose-finding cohorts. Subjects will be monitored for safety and the occurrence of DLTs. The trial follows a $3+3$ design for the dose-escalation steps. Three subjects will be enrolled sequentially per cohort. If the MTD is not reached in the planned dose-escalation cohorts, the RP2D will be based on the observed safety and immune-modulatory activity as a pharmacodynamic parameter supporting the RP2D decision.

To collect sufficient information regarding changes in immune-related parameters as a readout for pharmacodynamics of PRECIOUS-01 (eg, NY-ESO-1-specific CD8 ${ }^{+} \mathrm{T}$ cells, iNKT cell activation and NY-ESO-1 antibody responses in serum), the two highest dosing cohorts will be extended to a total of six subjects or the highest dosing cohort to a total of nine subjects, depending on the observed toxicity (cohort extension rules are listed in online supplemental table 1.

\section{Dose-escalation schedule and criteria}

The starting dose is $10 \%$ of the no observed adverse effect level in the non-clinical pivotal Good Laboratory Practice toxicology study. Doses will be escalated after a review of the safety data from the previous cohort by a 


\section{Box 1 Dose-limiting toxicity (DLT) criteria}

Injection site reaction $\geq$ grade 3 that persists more than 48 hours despite supportive care.

- Fever $\geq$ grade 3 lasting for more than 48 hours despite optimal medical intervention.

- Flu-like symptoms $\geq$ grade 3 lasting for more than 48 hours despite optimal medical intervention.

- Anaphylaxis $\geq$ grade 3 .

- Thromboembolic event $\geq$ grade 3.

- Fatigue $\geq$ grade 3 for $\geq 4$ days.

- Any other toxicity or combination of toxicities that would qualify as DLT in the opinion of the investigator.

The following toxicity is not considered a DLT:

- Isolated laboratory abnormalities $\geq$ grade 3 that resolve to $\leq$ grade 1 in $\leq 14$ days without clinical sequelae or need for therapeutic intervention.

dose steering board (DSB) on completion of the DLT observation period (ie, from the first administration up to and including 21 days after the first administration). Predefined DLT criteria will be used (box 1).

The trial follows a $3+3$ dose-finding design based on the occurrence of DLTs in subjects with NY-ESO-1-positive tumours. The dose-escalation parameters are as follows:

- Subjects are planned to be recruited in three fixed dose-finding cohorts. Doses are specified as follows: cohort 1 (low; $0.4 \mathrm{mg} / \mathrm{kg}$ PRECIOUS-01), cohort 2 (intermediate; $0.8 \mathrm{mg} / \mathrm{kg}$ PRECIOUS-01) and cohort 3 (high; $1.6 \mathrm{mg} / \mathrm{kg}$ PRECIOUS-01).

- Each subject will be observed for a minimum of 21 days (DLT observation period and cycle duration) before the next cohort is enrolled and may begin to receive PRECIOUS-01.

- As a safety precaution, in a cohort of three subjects, an initial sentinel subject in each cohort will receive PRECIOUS-01 and will be observed for 2 weeks before additional subjects in the cohort are dosed.

- If none of the three subjects in a dose cohort experience a DLT in the DLT observation period, enrolment of subjects into the next dose cohort will begin after review of the safety data by the DSB.

- If one out of the three subjects experiences a DLT, then up to a total of six evaluable subjects will be enrolled at the current dose level. Escalation will occur if no additional DLTs are seen in that cohort and after review of safety data by the DSB or data safety monitoring board (DSMB) (in case of $\geq$ grade 3 toxicity).

- If $\geq 2$ subjects in a dose cohort experience a DLT in the DLT observation period, enrolment at that dose level and dose escalation will stop and that dose will be considered to have exceeded the MTD.

- In case $\geq 2$ subjects in cohort three experience a DLT, this dose level will be de-escalated to $1.2 \mathrm{mg} / \mathrm{kg}$ and another three subjects will be enrolled in this de-escalated dose level. If one out of the three subjects experiences a DLT in this dose level, then up to a total of six evaluable subjects will be enrolled at the current $1.2 \mathrm{mg} / \mathrm{kg}$ dose level.

The MTD for this trial is defined as the dose level below the dose level at which $\geq 2$ subjects in a dose cohort experience a DLT within the DLT observation period. In case $\geq 2$ subjects in the first dose level (lowest dose level) experience a DLT, the dose levels will be de-escalated. If no DLT occurs at any of the dose levels tested, an RP2D of PRECIOUS-01 will be declared, if deemed appropriate, based on all available safety data, the immune modulation effect directly in tumour tissue and efficacy data.

\section{Dose modifications}

Dose modifications are not allowed, except in case of significant (grade 3 or 4) toxicities. Then, the following dose-modification criteria apply:

1. If the toxicity resolves to baseline or grade 1 (as defined by CTCAE V.5.0) in $\leq 14$ days after the intended start of the next treatment cycle, the treatment can continue to the next cycle at a dose of $50 \%$ of the starting dose of PRECIOUS-01 (for cohort 1, this will be based on a decision by the DSMB).

2. In case of a previous dose reduction or if toxicity does not resolve to baseline or grade 1 within 14 days after the intended start of the next treatment cycle, the subject must discontinue trial treatment.

\section{Eligibility criteria}

A summary of the eligibility criteria is listed in table 1 . The full list of eligibility criteria is specified in online supplemental table 2.

\section{Trial assessments}

The schedule of assessments is provided as online supplemental table 3 .

\section{Safety assessments}

Safety will be assessed according to NCI CTCAE V.5.0 using physical examination, weight, vital signs, Eastern Cooperative Oncology Group (ECOG) performance status, laboratory evaluations (haematology, coagulation, urinalysis and biochemistry), ECG, a pregnancy test in women of childbearing potential, recording of concurrent therapy and medications and treatment-emergent $\mathrm{AE} / \mathrm{SAE}$ reporting and follow-up.

\section{Immune-modulation assessments}

Mandatory tumour biopsies will be taken at baseline and after cycle 3 of PRECIOUS-01 (visit 11). The analyses on the composition and spatial heterogeneity of immune cell subsets in the biopsies will support the RP2D decision as part of this trials' primary objective. Moreover, heparin blood, serum and PAXgene blood will be collected (online supplemental table 3 ). Herein, the following analyses will be performed: occurrence and magnitude of functional responses of iNKT cells and T cells; serum cytokine levels (cytokine bead array); the specificity of $\mathrm{T}$ cells (multimer analysis); DC, iNKT cell and NK cell activation status and functionality (flow cytometry); cytokine responses (eg, 
Table 1 Key eligibility criteria

\section{Inclusion criteria}

1. Age $\geq 18$ years at time of signing informed consent.

2. Performance status $(E C O G \leq 1)$.

3. Estimated life expectancy of at least 6 months.

4. Histologically or cytologically confirmed advanced and/or metastatic solid tumour with progressive disease at baseline, for whom no standard treatment is available.

5. IHC-confirmed NY-ESO-1 positivity on (archival) tumour tissue (cut-off value: $>0 \%$ positive cells), per local laboratory guidelines (see online supplemental information).

6. Subject with evaluable disease per RECIST V.1.1.

7. Adequate haematologic, renal and liver function as defined by laboratory values performed within 14 days of start of treatment.

8. Previous therapy-derived toxicities should be resolved to grade $<2$ according to CTCAE V.5.0, with exceptions for alopecia.

9. Before registration, the subject's ability to give written informed consent according to International Council for Harmonisation Good Clinical Practice and national rules/local regulations.

\section{Exclusion criteria}

1. Second malignancy in the previous 2 years, except for adequately treated in situ carcinoma of the cervix uteri and basal or squamous cell carcinoma of the skin.

2. Clinical suspicion or radiological evidence of active brain metastases. Stable brain metastases without steroids are allowed.

3. Subjects with thromboembolic events within the past year.

4. Subjects on any other active anticancer therapy, except for gonadotropin-releasing hormone analogues or other hormonal or supportive care.

5. Subjects with major surgery within 4 weeks before initiating treatment or with minor surgical procedures within 7 days before initiating treatment or anticipating the need for major surgery during the trial.

6. Concomitant use of oral or intravenous immunosuppressive drugs. Inhaled, topical or intranasal steroids and adrenal replacement steroids $<10 \mathrm{mg} /$ day (prednisone equivalent) are permitted in the absence of autoimmune disease.

7. Uncontrolled infectious disease.

8. (Systemic) autoimmune disease, except type 1 diabetes mellitus, hypothyroidism after autoimmune thyroiditis and skin disorders (eczema and psoriasis).

9. History of clinically significant cardiovascular disease.

10. Serious bleeding and clotting condition(s).

11. Abnormal or clinically significant coagulation parameters at the discretion of the clinical investigator.

12. Any other conditions that may interfere with the planned treatment, affect subject compliance or place the subject at high risk from treatment-related complications.

13. History of severe allergic episodes and/or Quincke's oedema.

14. Prior allogeneic tissue/solid organ transplant, stem cell or bone marrow transplant.

15. Known hypersensitivity to any component of PRECIOUS-01.

16. Pregnant or lactating women.

CTCAE, Common Terminology Criteria for Adverse Events; ECOG, Eastern Cooperative Oncology Group ; IHC, immunohistochemistry; NY-ESO-1, New York Esophageal Squamous Cell Carcinoma-1; RECIST, Response Evaluation Criteria in Solid Tumors.

IFN- $\gamma$ ) in serum; anti-NY-ESO-1 antibody responses serum and mRNA levels for IFN-related genes (quantitative PCR). Furthermore, in cohort 2 and cohort 3 , a delayedtype hypersensitivity (DTH) skin test with PRECIOUS-01 will be performed at baseline and after the third administration of PRECIOUS-01. After 24 hours, skin biopsies will be taken. Herein, immune cell infiltrates will be characterised by IHC staining. DTH-derived T cells will be cultured, stimulated and evaluated for NY-ESO-1 specificity. Supernatants of these DTH cultures stimulated with NY-ESO-1-loaded target cells will be investigated by cytokine bead arrays on the presence of Th1-type/Th2-type cytokines.

\section{Efficacy assessments}

The preliminary clinical efficacy of PRECIOUS-01 will be analysed using the BOR rate. This exploratory efficacy endpoint will be assessed with CT scans at baseline and every 9 weeks thereafter. Imaging will be evaluated using the RECIST V.1.1 criteria. $^{28}{ }^{29}$ Partial and complete responses will be confirmed after 4 weeks.

\section{Statistical analysis plan}

The sample size is based on the determination of the MTD/RP2D. The MTD for this trial is defined as the dose level below the dose level at which $\geq 2$ subjects in a dose cohort experience a DLT (box 1) within the DLT observation period. All subjects receiving at least one dose of PRECIOUS-01 will be included in the evaluation of safety and assessment of clinical outcome. The design is not based on a formal sample size calculation and is not statistically powered for any objective. Therefore, descriptive statistics will be used to describe the safety, immune-modulatory activity and preliminary efficacy endpoints. All data will be documented using summary tables, figures and subject data listings. Continuous and categorical variables will be summarised using descriptive 
statistics (mean, SD, quartiles, minimum and maximum) and counts and percentages, respectively.

\section{ETHICS AND DISSEMINATION}

This trial will be conducted in conformance with the principles of the 'Declaration of Helsinki' (64th WMA General Assembly, Fortaleza, Brazil, October 2013) and fully adheres to the principles outlined in International Council for Harmonisation (ICH) E6-R2 or local law if it affords greater protection to the subject. Furthermore, as this trial is conducted in the European Union (EU)/ European Economic Area countries, it is compliant with the EU Clinical Trial Directive (2001/20/EC). Informed consent will be obtained in accordance with the Declaration of Helsinki, ICH Good Clinical Practice, the Data Protection Directive (Directive 95/46/EC) and local regulations. The trial is reviewed by the Dutch competent authority (CCMO; 27-07-2020) and approved by the medical research ethics committee (CMO ArnhemNijmegen; 02-09-2020) under registration number NL72876.000.20.

A summary of the results will be provided to the competent authority and medical research ethics committee within 1 year after the end of the trial. All subject records will be retained for 25 years following completion, termination or discontinuation of the clinical investigation. The results will be disseminated via (inter)national conferences and submitted for publication to a peerreviewed journal.

\section{PATIENT AND PUBLIC INVOLVEMENT}

Patients or the public were neither involved in the design nor will they be involved in this clinical trial's conduct, reporting or dissemination plans.

\section{DISCUSSION}

We propose a study protocol for a first-in-human phase I study with a new class of PLGA-based immunomodulatory nanoparticles-PRECIOUS-01-encapsulating tumor-antigen NY-ESO-1 and iNKT cell activator IMM60. PRECIOUS-01 tackles several hurdles present in traditional cellular immunotherapies: it is an off-the-shelf formulation, can be administered intravenously andthrough coencapsulation-can induce and direct a targeted immune response to NY-ESO-1-positive cancer cells. In addition, we hypothesise that PRECIOUS-01 has a favourable toxicity profile and that it can elicit multifunctional antitumour immune responses in patients with NY-ESO-1 expressing advanced cancers.

As in any biomarker-driven trial, the biomarkers' characteristics must be considered in the trials' design. In this protocol, the inclusion of subjects is based on the IHC determined NY-ESO-1 status on tumour tissue. Given the positivity threshold of $>0 \%$, NY-ESO-1 expression levels might vary between subjects, which can be considered a limitation. In this light, several factors need to be considered. First, the NY-ESO-1 expression follows a heterogeneous expression pattern in most tumour tissues, which on the one hand might limit the efficacy of a treatment, but on the other might propose an underestimation of actual NY-ESO-1 expression levels on inclusion. ${ }^{30}$ Second, expression of NY-ESO-1 on a tumour might increase in the course of the disease, as reflected by an increase in expression from primary to metastatic lesions ${ }^{3132}$ or increasing NY-ESO-1 antibody responses. ${ }^{33}$ Hence, archival tumour tissue might underestimate the NY-ESO-1 expression levels. Third, the correlation between NY-ESO-1 expression and the induction of antibody or T-cell responses is not clear-cut. For example, while in the phase I doseescalation trial with G305-a recombinant NY-ESO-1 protein vaccine-antibody and T-cell responses could be induced in patients with a high $(>70 \%)$ NY-ESO-1 expression, antibody or $\mathrm{CD} 4^{+} \mathrm{T}$-cell responses could also be induced in patients with a lower expression (eg, 15\% or $20 \%$ ). Moreover, though not powered for efficacy and only circumstantial evidence, two out of three patients with a NY-ESO-1 expression $\leq 20 \%$ showed radiological stable disease, while others with a higher expression demonstrated progressive disease. ${ }^{34}$ Similar findings of an absent correlation between NY-ESO-1 expression in tumour tissue and clinical activity were reported in another NY-ESO-1-based cancer vaccine trial. ${ }^{35}$ These observations might hypothetically be partly attributable to epitope spreading. ${ }^{36}$

In conclusion, with this phase I study protocol for a novel PLGA-based immunomodulatory nanomedicine, we strive to establish the safety and immunomodulatory effects of PRECIOUS-01.

\section{Author affiliations}

${ }^{1}$ Department of Tumor Immunology, Radboudumc, Nijmegen, The Netherlands ${ }^{2}$ Oncode Institute, Nijmegen, The Netherlands

${ }^{3}$ CATO SMS, Amsterdam, The Netherlands

${ }^{4}$ oncoKnow, Amsterdam, The Netherlands

${ }^{5}$ MRC Human Immunology Unit, Weatherall Institute of Molecular Medicine, Radcliffe Department of Medicine, University of Oxford, Oxford, UK

${ }^{6}$ Department of Oncology, University of Oxford, Oxford, UK

${ }^{7}$ Department of Medical Oncology, Radboudumc, Nijmegen, The Netherlands

${ }^{8}$ Department of Experimental Oncology and Molecular Medicine, Fondazione IRCCS Istituto Nazionale dei Tumori, Milan, Lombardia, Italy

${ }^{9}$ iOx Therapeutics, London, UK

Acknowledgements The authors would like to thank all PRECIOUS consortium partners for their contributions that ultimately led to this clinical trial's execution. Furthermore, the authors want to acknowledge the Methods in Clinical Cancer Research Workshop 2017 (Zeist, The Netherlands), where this protocol was (partially) developed.

Contributors CF conceptualised the research project. JHAC, IP and KG designed the clinical trial and wrote the clinical trial protocol. PBO, MM, WRG, NM, LR, IW, $\mathrm{CF}$ and IJMdV reviewed the protocol. JHAC wrote the manuscript. JHAC, IP, KG, UG, LR, CF, PBO and IJMdV reviewed and edited the manuscript. All authors read and approved the final manuscript.

Funding This work was supported by the European Union's Horizon 2020 Research and Innovation Programme under grant agreement number 686089.

Competing interests IW is an employee of i0x Therapeutics and has stock ownership. 
Patient consent for publication Not applicable.

Provenance and peer review Not commissioned; externally peer reviewed.

Supplemental material This content has been supplied by the author(s). It has not been vetted by BMJ Publishing Group Limited (BMJ) and may not have been peer-reviewed. Any opinions or recommendations discussed are solely those of the author(s) and are not endorsed by BMJ. BMJ disclaims all liability and responsibility arising from any reliance placed on the content. Where the content includes any translated material, BMJ does not warrant the accuracy and reliability of the translations (including but not limited to local regulations, clinical guidelines, terminology, drug names and drug dosages), and is not responsible for any error and/or omissions arising from translation and adaptation or otherwise.

Open access This is an open access article distributed in accordance with the Creative Commons Attribution Non Commercial (CC BY-NC 4.0) license, which permits others to distribute, remix, adapt, build upon this work non-commercially, and license their derivative works on different terms, provided the original work is properly cited, appropriate credit is given, any changes made indicated, and the use is non-commercial. See: http://creativecommons.org/licenses/by-nc/4.0/.

\section{ORCID iD}

Jeroen H A Creemers http://orcid.org/0000-0003-0371-8416

\section{REFERENCES}

1 Kumari A, Yadav SK, Yadav SC. Biodegradable polymeric nanoparticles based drug delivery systems. Colloids Surf $B$ Biointerfaces 2010;75:1-18.

2 Foged C, Sundblad A, Hovgaard L. Targeting vaccines to dendritic cells. Pharm Res 2002;19:229-38.

3 Waeckerle-Men Y, Groettrup M. Plga microspheres for improved antigen delivery to dendritic cells as cellular vaccines. Adv Drug Deliv Rev 2005;57:475-82.

4 Reichel D, Tripathi M, Perez JM. Biological effects of nanoparticles on macrophage polarization in the tumor microenvironment. Nanotheranostics 2019;3:66-88.

5 Feng X, Xu W, Li Z, et al. Immunomodulatory nanosystems. Adv Sci 2019;6:1900101.

6 Gnjatic S, Nishikawa $\mathrm{H}$, Jungbluth AA, et al. Ny-Eso-1: review of an immunogenic tumor antigen. Adv Cancer Res 2006;95:1-30.

7 Caballero OL, Chen Y-T. Cancer/Testis (CT) antigens: potential targets for immunotherapy. Cancer Sci 2009;100:2014-21.

8 Sharma P, Gnjatic S, Jungbluth AA, et al. Frequency of NY-ESO-1 and LAGE-1 expression in bladder cancer and evidence of a new NYESO-1 T-cell epitope in a patient with bladder cancer. Cancer Immun 2003;3:19.

9 Sugita $\mathrm{Y}$, Wada H, Fujita S, et al. Ny-Eso-1 expression and immunogenicity in malignant and benign breast tumors. Cancer Res 2004;64:2199-204.

10 Vaughan HA, Svobodova S, Macgregor D, et al. Immunohistochemical and molecular analysis of human melanomas for expression of the human cancer-testis antigens NY-ESO-1 and LAGE-1. Clin Cancer Res 2004;10:8396-404

11 Iura K, Maekawa A, Kohashi K, et al. Cancer-testis antigen expression in synovial sarcoma: NY-ESO-1, PRAME, MAGEA4, and MAGEA1. Hum Pathol 2017;61:130-9.

12 Gure AO, Chua R, Williamson B, et al. Cancer-testis genes are coordinately expressed and are markers of poor outcome in nonsmall cell lung cancer. Clin Cancer Res 2005;11:8055-62.

13 Tessari A, Pilla L, Silvia D, et al. Expression of NY-ESO-1, MAGE-A3, PRAME and WT1 in different subgroups of breast cancer: an indication to immunotherapy? Breast 2018;42:68-73.

14 IEDB. IEDB analysis resource: National Institute of allergy and infectious diseases, 2019. Available: http://tools.iedb.org/population/
15 Baumgaertner P, Costa Nunes C, Cachot A, et al. Vaccination of stage III/IV melanoma patients with long NY-ESO-1 peptide and $\mathrm{CpG}-\mathrm{B}$ elicits robust $\mathrm{CD} 8^{+}$and $\mathrm{CD} 4^{+} \mathrm{T}$-cell responses with multiple specificities including a novel DR7-restricted epitope. Oncoimmunology 2016;5:e1216290.

16 Chen J-L, Dawoodji A, Tarlton A, et al. Ny-Eso-1 specific antibody and cellular responses in melanoma patients primed with NY-ESO-1 protein in ISCOMATRIX and boosted with recombinant NY-ESO-1 fowlpox virus. Int J Cancer 2015;136:E590-601.

17 Odunsi K, Matsuzaki J, Karbach J, et al. Efficacy of vaccination with recombinant vaccinia and fowlpox vectors expressing NY-ESO-1 antigen in ovarian cancer and melanoma patients. Proc Natl Acad Sci U S A 2012;109:5797-802.

18 Sabbatini P, Tsuji T, Ferran L, et al. Phase I trial of overlapping long peptides from a tumor self-antigen and poly-ICLC shows rapid induction of integrated immune response in ovarian cancer patients. Clin Cancer Res 2012;18:6497-508.

19 Jukes J-P, Gileadi U, Ghadbane H, et al. Non-glycosidic compounds can stimulate both human and mouse iNKT cells. Eur J Immunol 2016;46:1224-34.

20 Vivier E, Ugolini S, Blaise D, et al. Targeting natural killer cells and natural killer T cells in cancer. Nat Rev Immunol 2012;12:239-52.

21 Dölen Y, Gileadi U, Chen J-L, et al. Plga nanoparticles Coencapsulating NY-ESO-1 peptides and IMM60 induce robust CD8 and CD4 T cell and B cell responses. Front Immunol 2021;12:641703.

22 Dölen $\mathrm{Y}$, Valente $\mathrm{M}$, Tagit $\mathrm{O}$, et al. Nanovaccine administration route is critical to obtain pertinent iNKt cell help for robust anti-tumor T and B cell responses. Oncoimmunology 2020;9:1738813.

23 Bae E-A, Seo H, Kim I-K, et al. Roles of NKT cells in cancer immunotherapy. Arch Pharm Res 2019;42:543-8.

24 Cerundolo V, Silk JD, Masri SH, et al. Harnessing invariant NKT cells in vaccination strategies. Nat Rev Immunol 2009;9:28-38.

25 Fujii S-I, Shimizu K. Immune Networks and Therapeutic Targeting of iNKT Cells in Cancer. Trends Immunol 2019;40:984-97.

26 McEwen-Smith RM, Salio M, Cerundolo V. The regulatory role of invariant NKT cells in tumor immunity. Cancer Immunol Res 2015;3:425-35

27 Yamashita K, Arimoto A, Nishi M, et al. Application of iNKT celltargeted active immunotherapy in cancer treatment. Anticancer Res 2018;38:4233-9.

28 Services USDoHaH, Administration FaD, Excellence OCo. Clinical trial endpoints for the approval of cancer drugs and biologics guidance for industry, 2018.

29 Eisenhauer EA, Therasse P, Bogaerts J, et al. New response evaluation criteria in solid tumours: revised RECIST guideline (version 1.1). Eur J Cancer 2009;45:228-47.

30 Thomas R, Al-Khadairi G, Roelands J, et al. Ny-Eso-1 based immunotherapy of cancer: current perspectives. Front Immunol 2018;9:947.

31 Aung PP, Liu Y-C, Ballester LY, et al. Expression of New York esophageal squamous cell carcinoma-1 in primary and metastatic melanoma. Hum Pathol 2014;45:259-67.

32 Park TS, Groh EM, Patel K, et al. Expression of MAGE-A and NY-ESO-1 in primary and metastatic cancers. J Immunother 2016;39:1-7.

33 Oshima Y, Shimada H, Yajima S, et al. NY-ESO-1 autoantibody as a tumor-specific biomarker for esophageal cancer: screening in 1969 patients with various cancers. J Gastroenterol 2016;51:30-4.

34 Mahipal A, Ejadi S, Gnjatic S, et al. First-in-human phase 1 dose-escalating trial of G305 in patients with advanced solid tumors expressing NY-ESO-1. Cancer Immunol Immunother 2019;68:1211-22.

35 Dhodapkar MV, Sznol M, Zhao B, et al. Induction of antigen-specific immunity with a vaccine targeting NY-ESO-1 to the dendritic cell receptor DEC-205. Sci TransI Med 2014;6:232ra51.

36 Brossart $P$. The role of antigen spreading in the efficacy of immunotherapies. Clin Cancer Res 2020;26:4442-7. 